\title{
HERAPDF1.5LO PDF Set with Experimental Uncertainties
}

\author{
A M Cooper-Sarkar* \\ Oxford University, $U K$ \\ E-mail: a.cooper-sarkarl@physics.ox.ac.uk
}

LO PDFs are useful for Monte Carlo generators based on LO matrix elements plus parton showers. This contribution presents the HERAPDF1.5 PDF set evolved to LO in $\alpha_{s}$

XXII. International Workshop on Deep-Inelastic Scattering and Related Subjects,

28 April - 2 May 2014

Warsaw, Poland

*Speaker. 


\section{Introduction}

Parton densities evolved to leading order (LO) in $\alpha_{s}$ are essential for the proper simulation of parton showers (PS) and underlying event properties in LO+PS Monte Carlo (MC) event generators.

In 2015 the LHC will restart with upgraded proton beam energies, these higher energies will extend the kinematic reach of the LHC to lower values of the Bjorken- $x$ variable. The HERAPDF gives special emphasis to the low- $x$ region and thus it is particularly suited to use in the new tunes that are being developed for the simulation of the underlying event, minimum bias events and pile-up for LHC Run-II.

In this note the HERAPDF1.5 LO set is presented based on the same settings as used for the HERAPDF1.5 NLO PDF set[1], with the exception of the use of the LO DGLAP splitting kernel and, correspondingly, of a different value for the strong coupling constant.

\section{Technical Description of the HERAPDF1.5 LO PDF set}

The framework used in this QCD analysis is based on the HERAFitter project [2], with evolution code as implemented in the QCDNUM package [3]. The results were cross checked by an independent framework referred to as the ZEUS Fitter [4]. The QCD fit settings are adopted from the previous HERAPDF fits to preliminary combined H1 and ZEUS HERA I+II data of inclusive deep-inelastic scattering used to extract HERAPDF1.5 NLO [1] and NNLO PDF [5] sets. The experimental uncertainties of data are treated in the same way as in the HERAPDF1.5 NLO and NNLO fits. The PDFs parametrised at the starting scale of the evolution ${ }^{1}$ of $Q_{0}^{2}=1.9 \mathrm{GeV}^{2}$ are the valence distributions $x u_{v}$ and $x d_{v}$, the gluon distribution $x g$, and the $x \bar{U}$ and $x \bar{D}$ distributions, where $x \bar{U}=x \bar{u}, x \bar{D}=x \bar{d}+x \bar{s}$. The following functional form is used to parametrise them:

$$
\begin{aligned}
x u_{v}(x) & =A_{u_{v}} x^{B_{u_{v}}}(1-x)^{C_{u_{v}}}\left(1+E_{u_{v}} x^{2}\right) \\
x d_{v}(x) & =A_{d_{v}} x^{B_{d_{v}}}(1-x)^{C_{d_{v}}} \\
x \bar{U}(x) & =A_{\bar{U}} x^{B_{\bar{U}}}(1-x)^{C_{\bar{U}}} \\
x \bar{D}(x) & =A_{\bar{D}} x^{B_{\bar{D}}}(1-x)^{C_{\bar{D}}} \\
x g(x) & =A_{g} x^{B_{g}}(1-x)^{C_{g}} .
\end{aligned}
$$

where the normalization parameters $\left(A_{u_{v}} ; A_{d_{v}} ; A_{g}\right)$ are constrained by quark counting and momentum sum rules. The $B$ exponents for the quark sea and valence distributions, respectively, are set equal, $B_{\bar{U}}=B_{\bar{D}}$ and $B_{u_{v}}=B_{d_{v}}$. The strange quark distribution at the starting scale is assumed to be a constant fraction of $\bar{D}, x \bar{s}=f_{s} x \bar{D}$, chosen to be $f_{s}=0.31$ such that $\bar{s} \approx \bar{d} / 2$. In addition, to ensure that $x \bar{u}=x \bar{d}$ as $x \rightarrow 0, A_{\bar{U}}=A_{\bar{D}}\left(1-f_{s}\right)$. This yields 10 free parameters the same as HERAPDF1.5NLO. Note that the LO gluon has no term which allows it to become negative at low $x$ and low scale.

The PDFs are evolved using the DGLAP evolution equations at LO with the renormalisation and factorisation scales set to the squared momentum transfer of the NC or CC interaction, $Q^{2}$.

\footnotetext{
${ }^{1}$ chosen to be below the charm mass threshold as required by the QCDNUM package
} 
The value for $\alpha_{s}\left(M_{Z}\right)$ has been chosen to be 0.13 , which yields the best level of agreement between data and the fit $^{2}$.

As for previous HERAPDF PDF sets, the analysis is performed accounting for the charm and beauty quark masses in the Thorne-Roberts (TR) Variable Flavour Number Scheme [8]. In this scheme the leading order for the calculation of the longitudinal structure function $F_{L}$ is defined as its leading non-zero contribution, i.e. at $\mathrm{O}\left(\alpha_{s}\right)$. . Note that $F_{L}=0$ at zeroth order in $\alpha_{s}$ and good fits to the data cannot be obatined.

The experimental uncertainties on the PDFs are determined using the $\Delta \chi^{2}=1$ criterion leading to uncertainties with a confidence level of $68 \%$. The $\chi^{2}$ is defined as in [9]:

$$
\chi^{2}=\sum_{i} \frac{\left[\mu_{i}-m_{i}\left(1-\sum_{j} \gamma_{j}^{i} b_{j}\right)\right]^{2}}{\delta_{i, \mathrm{unc}}^{2} m_{i}^{2}+\delta_{i, \mathrm{stat}}^{2} \mu_{i} m_{i}\left(1-\sum_{j} \gamma_{j}^{i} b_{j}\right)}+\sum_{j} b_{j}^{2}
$$

where $m_{i}$ is the theoretical prediction and $\mu_{i}$ is the measured cross section at point $i,\left(Q^{2}, x, s\right)$ with the relative statistical and uncorrelated systematic uncertainty $\delta_{i, \text { stat }}, \delta_{i, \text { unc }}$, respectively. The values $\gamma_{j}^{i}$ denote the relative correlated systematic uncertainties and $b_{j}$ their shifts with a penalty term $\sum_{j} b_{j}^{2}$ added.

\section{QCD Fit Results and Comparisons}

The LO QCD fit to the preliminary combined H1 and ZEUS HERA I+II data[1] yields a reasonable total $\chi^{2}$ of 762 for 664 degrees of freedom, slightly worse than the NLO $\chi^{2}$ of 736 . The choice of $\alpha_{s}\left(M_{Z}\right)=0.13$ was motivated through a scan procedure, where the data were refit with other choices of the strong coupling and the best value in terms of fit quality was chosen. The resulting PDF distribution plots for the starting scale as well as for momentum transfer 10000 $\mathrm{GeV}^{2}$ can be found in Figure 1. They are presented together with uncertainty bands reflecting the experimental uncertainties.

Good agreement of the LO fit with all four differential cross section measurements (neutral and charged currents, $e^{+} p$ and $e^{-} p$ ) is achieved. This is illustrated for the neutral current $e^{+} p$ differential measurements in Figure 2.

The LO PDF set has been formatted to match the LHAPDF style, similarly to what was done for the HERAPDF1.5 NLO set, compatible with the LHAPDFv5 and LHAPDFv6 grid style. The LHAPDF grid contains 21 members with member 0 representing the central fit, while members $1-20$ correspond to the experimental uncertainties on the PDFs. The 20 error PDFs should be treated two by two as the up and down excursions for each of the 10 eigenvectors defined by the number of free PDF parameters in the fit, such that the symmetric error is calculated as the quadratic sum of the difference between the up and down eigenvectors divided by two. If asymmetric errors are desired equation 43 of [10] may be used.

\footnotetext{
${ }^{2}$ This value agrees with the value used by the CTEQ LO set [6] of $\alpha_{S}\left(M_{Z}\right)=0.12978$, which is different from the value chosen by the MSTW LO set [7] of $\alpha_{S}\left(M_{Z}\right)=0.136$.
} 

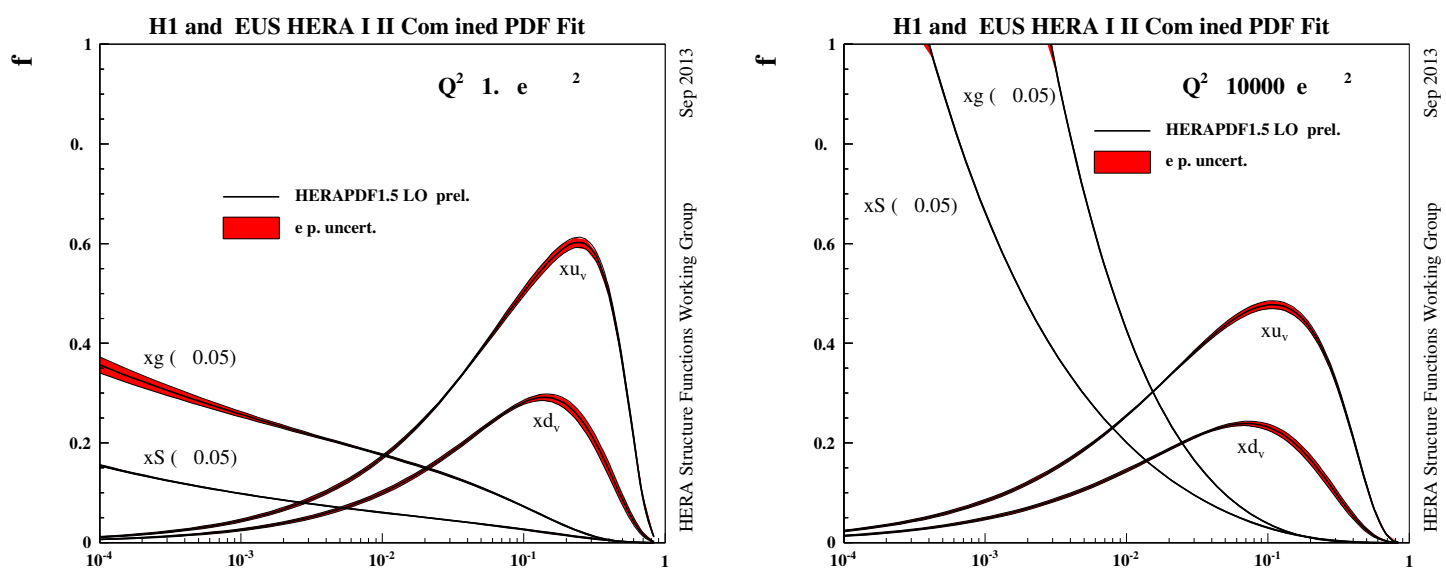

Figure 1: Summary of LO PDFs at $Q^{2}=1.9 \mathrm{GeV}^{2}$ (left) and at $Q^{2}=M_{W}^{2} \mathrm{GeV}^{2}$ (right)

\section{Use of HERAPDF1.5LO in Monte-Carlos}

Figure 3 shows the inclusive jet spectrum for central and forward jets as measured by CMS compared to predictions using PYTHIA and the Z2* tune with HERAPDF1.5LO and with the default CTE6L. the predictions are shown with and without simulation of Multi-Parton Interactions. Figure 4 shows similar predictions for the energy flow in minimum bias events and in di-jet events. Figure 5 shows similar predictions for the transeverse momentum and charge density in underlying events compared to ATLAS data. HERAPDF1.5LO describes the data as well as, and in the case of the underlying event better than, CTEQ6L even thogh the Z2* tune was tuned to CTEQ6L.

\section{Summary}

A HERAPDF1.5 LO PDF set has been extracted based on the preliminary HERA I+II H1 and ZEUS combined NC and CC measurements, providing experimental uncertainties. The fit describes the DIS data well. Predictions for soft-physics at the LHC are competitive with, or better than, those of currently used PDFs like CTEQ6L.

\section{Acknowledgements}

I thank Hannes Jung for the figures in Section 4.

\section{References}

[1] A. M. Cooper-Sarkar [ZEUS and H1 Collaborations], arXiv:1112.2107 [hep-ph]. V. Radescu et al. [H1 and ZEUS Collaborations], arXiv:1308.0374 [hep-ex].

[2] F.D. Aaron et al. [H1 and ZEUS Collaborations], JHEP 1001:109 (2010); [arXiv:0911.0884 [hep-ex]]. F.D. Aaron et al. [H1 Collaboration], Eur. Phys. J. C 64 (2009) 561; [arXiv:0904.3513 [hep-ex]]. 


\section{H1 and EUS}

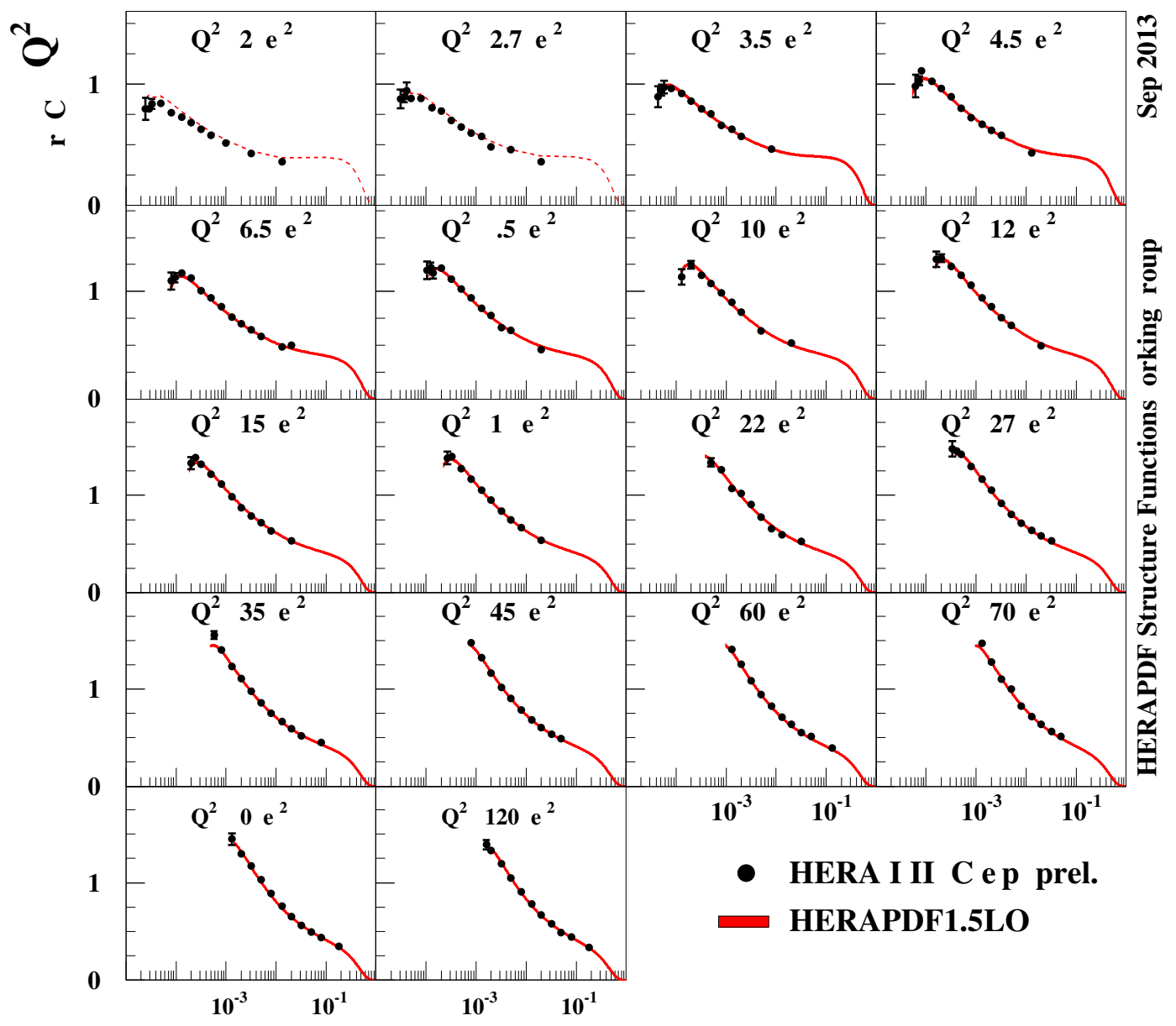

Figure 2: Neutral current $e^{+} p$ differential cross section measurements (for lower $Q^{2}$ bins) compared to predictions based on the HERAPDF1.5 LO PDF set with experimental uncertainties included in the predictions. The dashed line indicates predictions for kinematic regions not included in the fit.

[3] M. Botje, arXiv:1005.1481 [hep-ph].

[4] S. Chekanov et al. [ZEUS Collaboration], Eur. Phys. J. C 42 (2005) 1; [hep-ph/0503274].

[5] V. Radescu [H1 and ZEUS Collaborations], arXiv:1107.4193 [hep-ex].

[6] H.L. Lai et al., Eur. Phys. J. C 12 (2000) 375; [hep-ph/9903282].

[7] A.D. Martin, W.J. Stirling, R.S. Thorne and G. Watt Eur. Phys. J. C 63 (2009) 189; [arXiv:0901.0002 [hep-ph]].

[8] R.S. Thorne, R.G. Roberts, Phys. Rev. D57 (1998) 6871; [hep-ph/9709442].

R.S. Thorne, Phys. Rev. D73 (2006) 054019; [hep-ph/0601245]. 

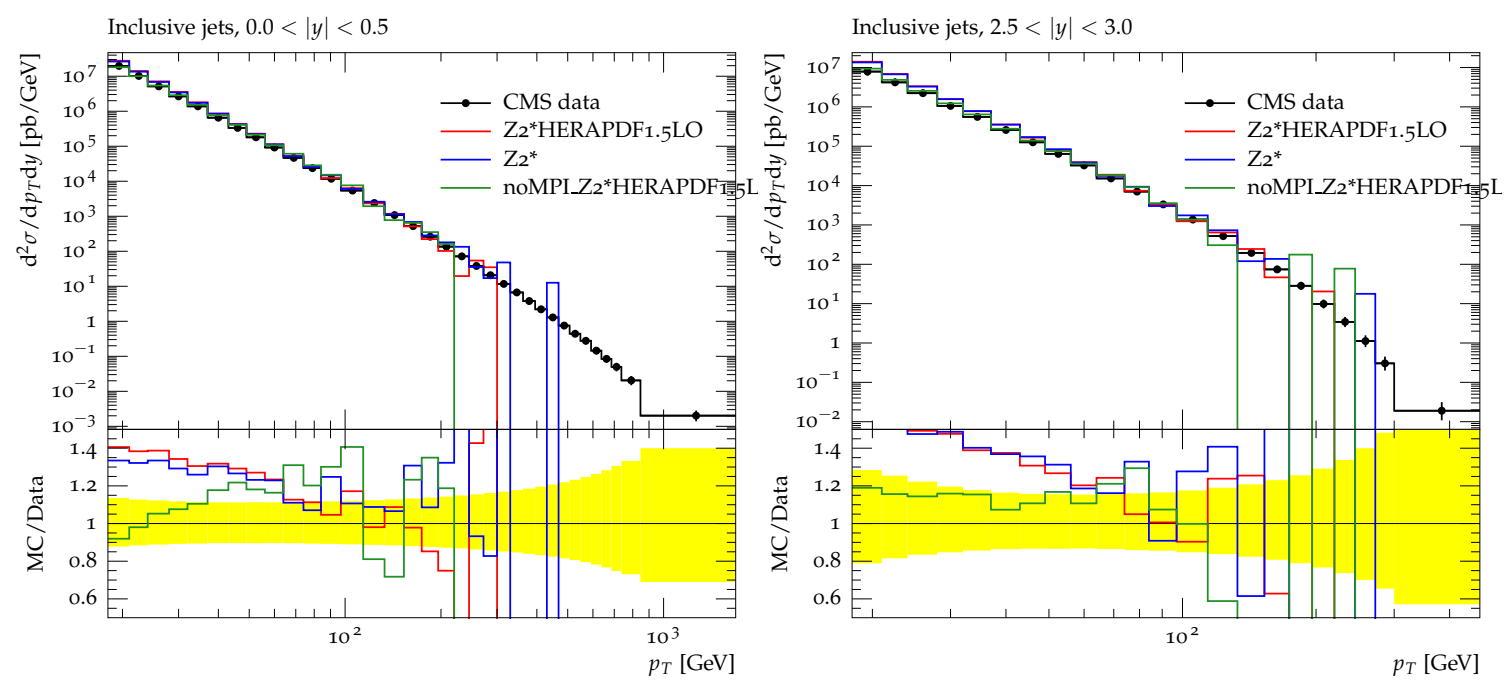

Figure 3: Comparison of predictions from PYTHIA using HERAPDFLO1.5 and CTEQ6L for the $p_{t}$ spectrum of inclusive jets at central rapdity (left) and forward rapidity (right) to CMS data
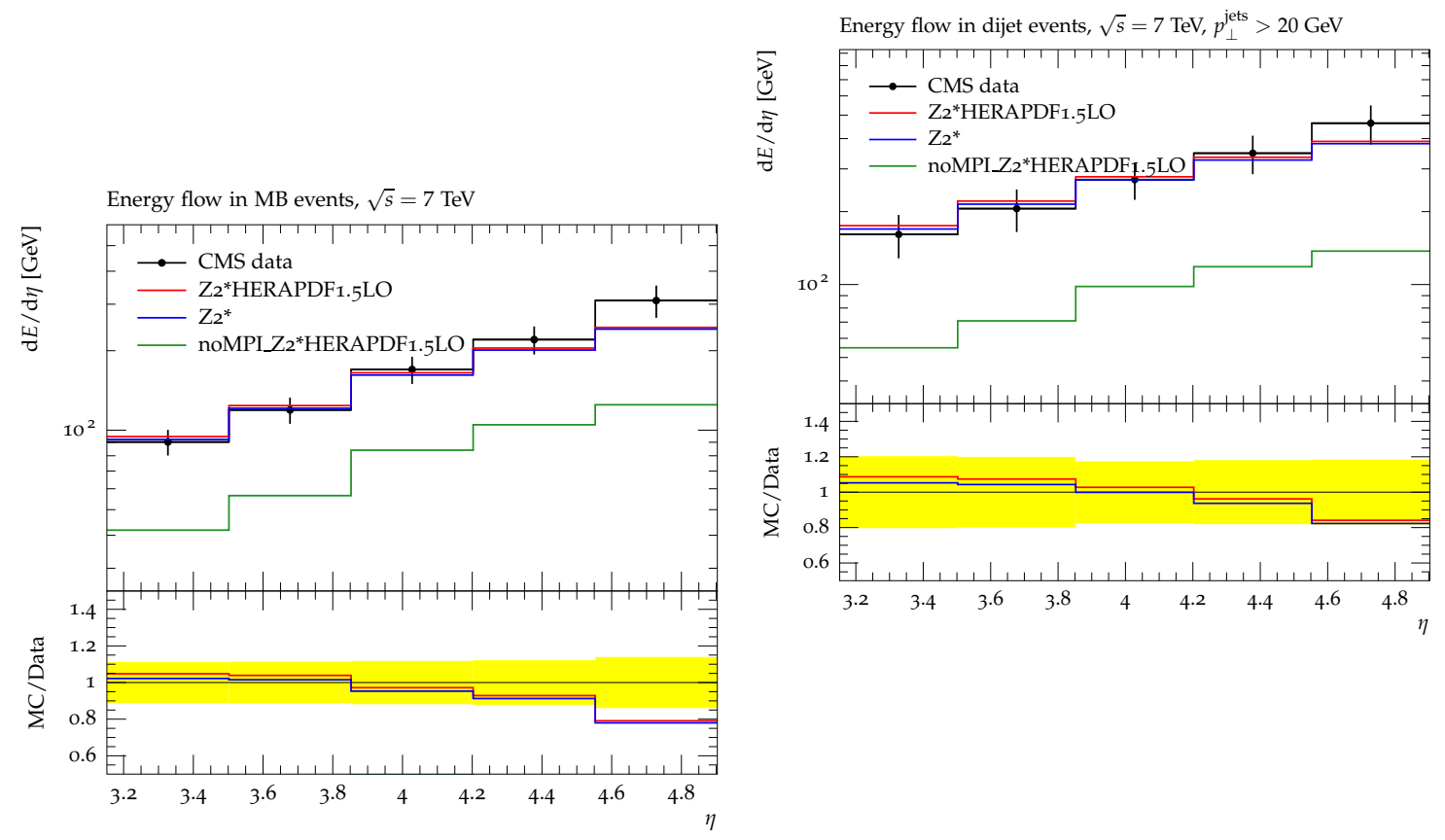

Figure 4: Comparison of predictions from PYTHIA using HERAPDFLO1.5 and CTEQ6L for the energy flow in Minimum Bias events (left) and dijets (right) to CMS data 

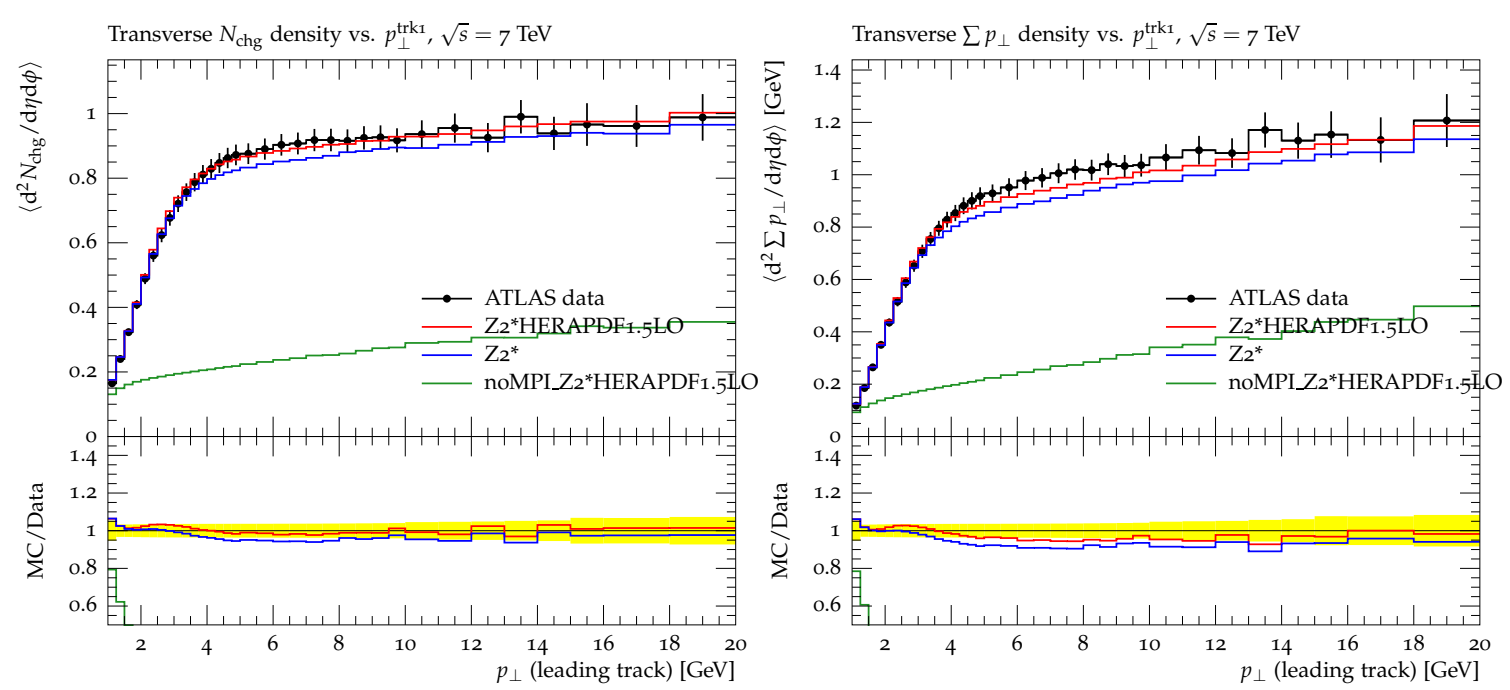

Figure 5: Comparison of predictions from PYTHIA using HERAPDFLO1.5 and CTEQ6L for the transverse momentum (left) and charge density (right) in underlying events to ATLAS data

[9] F. D. Aaron et al. [ H1 Collaboration], Eur. Phys. J. C 71 (2011) 1579; [arXiv:1012.4355 [hep-ex]].

[10] J.M. Campbell, J.W. Huston and W.J. Stirling, Rept. Prog. Phys. 70 (2007) 89; [hep-ph/0611148]. 